\title{
Blood Flow and Distribution in the Canine Pancreas
}

\author{
James A. KNOL, M.D., William E. Strodel, M.D., AND Frederic E. ECKhauser, M.D. \\ Department of Surgery, Ann Arbor Veterans Administration Medical Center, and The University \\ of Michigan Medical Center, Ann Arbor, Michigan 48109-0331
}

Submitted for publication February 4, 1986

\begin{abstract}
Because of a tripartite arterial inflow, accurate determination of canine pancreatic blood flow $\left(Q_{\mathrm{P}}\right)$ in experimental studies remains problematic. Assessment of blood flow using a single electromagnetic flow probe on the anterior pancreaticoduodenal artery (APDA) was compared to the radiolabeled microsphere method. Distribution of $Q_{\mathrm{P}}$ was based on microsphere density. $Q_{\mathrm{P}}$ determined simultaneously with the flow probe technique and the microsphere method were $86 \pm 17$ and $23 \pm 8 \mathrm{ml} / \mathrm{min}$, respectively, $(P<0.05)$. Following occlusion of the splenic artery $(\mathrm{SA})$ and the posterior pancreaticoduodenal artery (PPDA), $Q_{\mathrm{P}}$ measured by the flow probe increased to $94 \pm 27 \mathrm{ml} / \mathrm{min}$ (NS) and decreased to $19 \pm 4 \mathrm{ml} / \mathrm{min}$ (NS) using microspheres. Intrapancreatic distribution of $Q_{\mathrm{P}}$ was not significantly altered by occlusion of the SA and PPDA. Intrapancreatic arterial collateral is adequate to maintain blood flow to the entire pancreas even when arterial inflow is restricted to the APDA. Flow probe determinations of $Q_{\mathrm{P}}$ are artifactually high because they include flow to the duodenum and may also be subject to methodologic error. (c) 1987 Academic Press, Inc.
\end{abstract}

\section{INTRODUCTION}

Despite the frequent use of pancreatic blood flow measurement in experimental studies involving the canine pancreas, there remains a great deal of uncertainty as to the validity of some of the currently accepted methods for estimating pancreatic blood flow. Arterial supply to the canine pancreas in tripartite. The largest inflow vessel is the anterior pancreaticoduodenal artery (APDA), a terminal branch of the gastroduodenal artery, which enters the head of the pancreas and arborizes over the pancreatic head, uncinate process, and duodenum. Branches of the splenic artery (S $\Lambda$ ) arborize to supply the body and tail of the gland. The third and smallest source of arterial inflow is the posterior pancreaticoduodenal artery (PPDA) which arises from the anterior mesenteric artery and supplies the tip of the uncinate process and a portion of the distal duodenum. Functional collateralization among these three pancreatic arterial distributions has never been adequately demonstrated. Nevertheless, some investigators have maintained that canine pancreatic blood flow can be fully maintained by the APDA and that measurement of APDA flow after ligation of the PPDA and pancreatic branches of the SA is representative of total pancreatic blood flow [1-3]. On the contrary, others have suggested that the three arterial distribution areas are nearly separate and feel that estimating total pancreatic blood flow based on measurement of APDA flow after ligation of all other pancreatic arterial inflow is unsound $[4,5]$.

In order to examine the adequacy of canine intrapancreatic arterial collateral, pancreatic blood flow distribution was measured using radiolabeled carbonized plastic microspheres, both with all arterial inflow open and with only the APDA open. The distribution of flow was not significantly different when based on the single artery, but total pancreatic blood flow was somewhat reduced. Comparison with simultaneous electromagnetic flow probe measurement of APDA flow revealed major discrepancies in valucs obtained by the two measurement methods.

\section{MATERIALS AND METHODS}

Eight conditioned mongrel dogs weighing 21 to $32 \mathrm{~kg}$ were anesthetized with intrave- 
nous pentobarbital sodium $30 \mathrm{mg} / \mathrm{kg}$, intubated, and ventilated on room air using a Harvard volume ventilator. Ventilatory adequacy was monitored with arterial blood gas determinations. Anesthesia was maintained with supplemental doses of pentobarbital sodium, with the last dose given at least $30 \mathrm{~min}$ before any experimental observations. Body temperature was maintained at $38 \pm 0.5^{\circ} \mathrm{C}$ with a warming mattress and a heat lamp. A femoral arterial cannula was positioned with its tip in the distal abdominal aorta for arterial pressure monitoring and for withdrawal of microsphere reference samples. Lactated Ringer's solution was infused through a femoral venous cannula at $5 \mathrm{ml} / \mathrm{kg} / \mathrm{hr}$ to maintain intravascular volume. A right carotid artery catheter was positioned with its tip in the left ventricle for microsphere injection. A thermistor tip Swan-Ganz catheter was introduced through the external jugular vein and positioned in the pulmonary artery for determination of central venous pressure, pulmonary artery wedge pressure, and thermodilution cardiac output. Through a midline abdominal incision the gastroduodenal artery was isolated and encircled with a calibrated electromagnetic flow probe. The right gastroepiploic artery was ligated distal to all pancreatic branches. The SA was encircled with snares proximal and distal to its pancreatic branchcs, and all nonpancreatic branches between the snares were ligated. The PPDA was encircled with a snare proximal to its pancreatic branches. The model is depicted in Fig. 1.

After a 30-min stabilization period, cardiac output, arterial pressure, central venous pressure, and pulmonary artery wedge pressure were determined. Five minutes later, pancreatic blood flow was measured using microspheres according to the method of Heymann et al. [6]. Carbonized plastic ${ }^{141} \mathrm{Ce}$ and ${ }^{85} \mathrm{Sr}$-labeled microspheres $15 \pm 3 \mu \mathrm{m}$ in diameter were injected into the left ventricle over $20 \mathrm{sec}$, including two flushes with warmed normal saline. Beginning with the start of injection, blood was withdrawn from the distal aortic catheter at $30 \mathrm{ml} / \mathrm{min}$ for 1

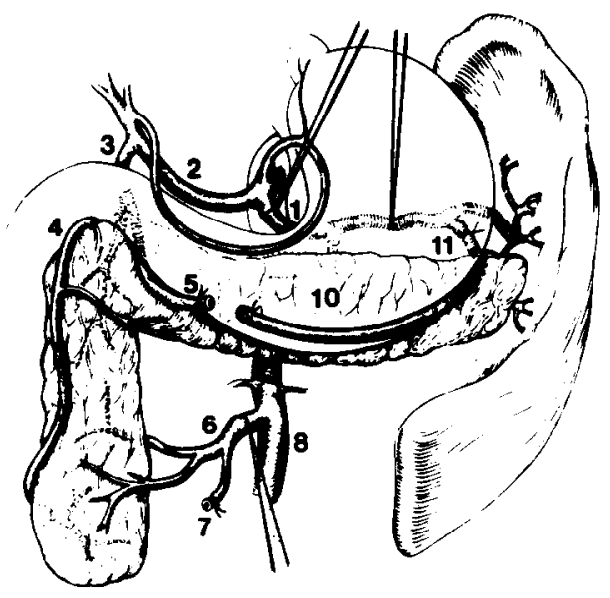

FIG. 1. Model for determination of pancreatic blood distribution with splenic artery (1) and posterior pancreaticoduodenal artery (6) snares for occlusion. Flow probe was placed on the gastroduodenal artery (3).

min. Doses of microspheres were calculated to yield 400 microspheres per tissue sample. Five minutes after the first microsphere injection, the snares on the SA and PPDA were tightened and the second microsphere injection was performed; 3 min later the snares were again loosened. In two dogs the snares on the PPDA and SA were not tightened at the time of the second injection of microspheres, and these dogs were excluded from the analysis of blood flow distribution of the APDA. APDA electromagnetic probe blood flow readings were recorded continuously throughout the period of microsphere injections.

Following measurement of postinjection parameters, the dog was sacrificed using intravenous barbiturate overdose. The entire pancreas and the parapancreatic duodenum were excised, drained of blood, and weighed separately. Reference blood specimens were anticoagulated with a small amount of heparin, measured for volume, and assayed for radioactivity. The entire pancreas and the parapancreatic duodenum to just below the entrance of the pancreatic duct were measured and sectioned as in Fig. 2. Tissue was assayed for radioactivity on a Beckman automated gamma counter and blood flow was calculated for each specimen [6]. 


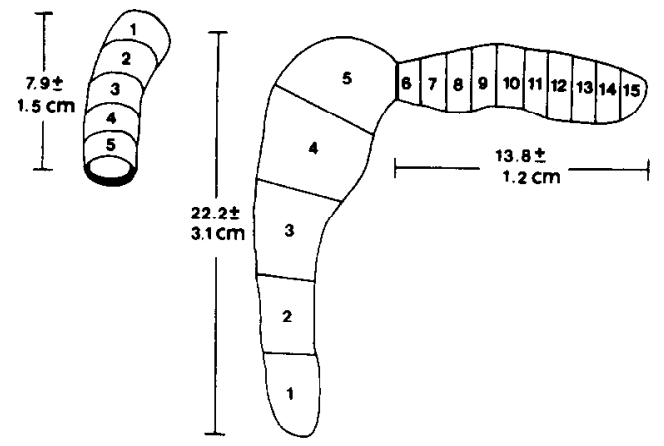

FIG. 2. Pattern for subdivision of pancreas and duodenum for determination of blood flow distribution.

Animal care, use, and handling followed the guidelines and standards of the Veterans Administration, the American Association for the Accreditation of Laboratory Animal Care, and the Michigan Standard Care Procedures and were reviewed and approved by the Committee on Animal Studies at the Ann Arbor Veterans Administration Medical Center.

Data were compiled on the Michigan Terminal System and analyses utilized paired Student's $t$ tests for comparisons of changes in hemodynamic parameters and in total pancreatic and duodenal blood flow within each of the microsphere and electromagnetic flow probe methods. Unpaired Students $t$ tests were performed in comparisons of total pancreatic and duodenal blood flows between methods. One-way analysis of variance with comparisons (or the KruskalWallis test when data did not fit the normal distribution) was applied to the distribution of blood flow across the 15 pancreatic segments and the 5 duodenal segments. Twoway analysis of variance was used to compare segmental distribution changes due to occluding the PPDA and SA. Data are presented as means $\pm \mathrm{SD}$. Statistical significance assumes that $P<0.05$.

\section{RESULTS}

All animals survived the experimental period. There were no gross appearance changes in either the pancreas or the duode- num in these animals during the course of the experiment.

Over the period of microsphere injections cardiac index fell 3\% from 2.34 to 2.26 liters/min, pulmonary wedge pressure fell $4 \%$ from 7.3 to $7.0 \mathrm{~mm} \mathrm{Hg}$, and central venous pressure fell $4 \%$ from 4.7 to $4.5 \mathrm{~mm} \mathrm{Hg}$. These changes are not statistically significant for any parameter. Mean arterial pressure rose $5 \%$ from 126 to $132 \mathrm{~mm} \mathrm{Hg}$ (NS) over the same period. Systemic temperature remained unchanged at $38.1^{\circ} \mathrm{C}$.

Pancreatic head-uncinate length averaged $22.2 \pm 3.1 \mathrm{~cm}$ (range, $17-27 \mathrm{~cm}$ ) and weight averaged $30.6+7.0 \mathrm{~g}$ (range, 23.7-42.8 g). Pancreatic body-tail length averaged 13.8 $\pm 1.2 \mathrm{~cm}$ (range, $11.5-15.0 \mathrm{~cm}$ ) and weight averaged $18.1 \pm 3.3 \mathrm{~g}$ (range, 13.1-22.0 g). Total pancreatic weight was $48.7 \pm 9.8 \mathrm{~g}$ (range, 36.8-63.7 g). Parapancreatic duodenal length was $7.9 \pm 1.5 \mathrm{~cm}$ (range, 6-11 $\mathrm{cm}$ ) and weight was $23.9 \pm 4.3 \mathrm{~g}$ (range, 17.5-29.0 g).

Distribution studies of pancreatic blood flow $(\mathrm{ml} / \mathrm{min} / 100 \mathrm{~g})$ in the dogs with intact arterial inflow to the pancreas showed no evidence of significant blood flow differences across the gland (Fig. 3). Blood flow tended to be less at the tip of the uncinate and in the tail of the pancreas. Occlusion of inflow through the SA and PPDA was associated with a fall in blood flow in all pancreatic segments (NS). Distribution of blood flow throughout the pancreas was not affected by

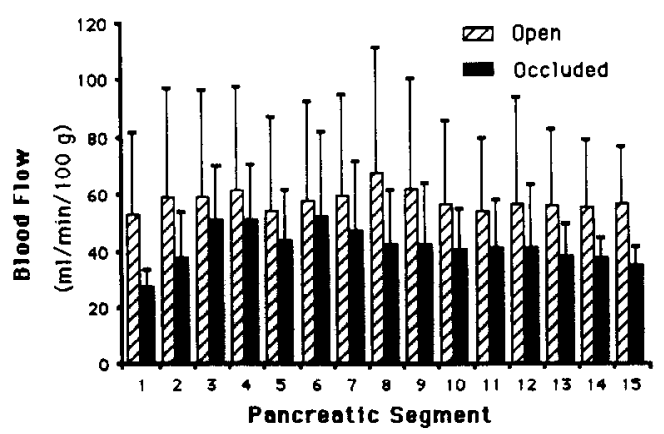

FIG. 3. Distribution of pancreatic blood flow with the SA and PPDA open and occluded. Segments correspond to those in Fig. 2. 


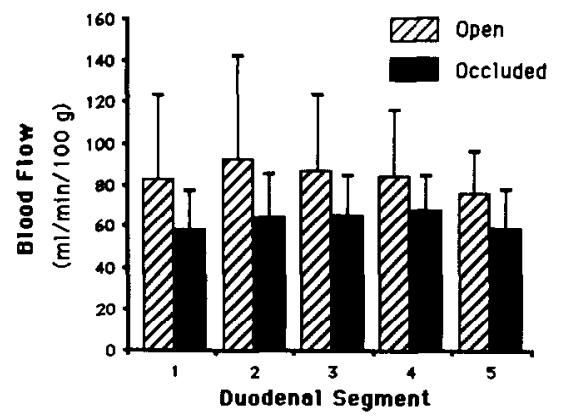

FIG. 4. Distribution of blood flow in the parapancreatic duodenal segment with the SA and PPDA open and occluded. Segments correspond to those in Fig. 2.

occluding the SA and the PPDA and basing the entire arterial inflow on the APDA.

Blood flow through the parapancreatic duodenal segment was higher than through the adjacent pancreas (Fig. 4). Flow was uniform across the duodenal segment. Although clamping the SA and PPDA produced a slight decrease in flow, the magnitude was not significant; occluding those vessels did not affect the distribution of flow across the duodenal segment.

Flow rates determined by an electromagnetic flow probe around the APDA were 86.8 $\pm 16.6 \mathrm{ml} / \mathrm{min}$ in animals with an intact pancreatic arterial supply and $93.8 \pm 27.5$ $\mathrm{ml} / \mathrm{min}$ after occlusion of the SA and PPDA. This increase in APDA flow was not statistically significant. The rise in APDA flow was rapid and coincided with occlusion of the PPDA and SA.

Comparisons of blood flows using the microsphere versus the electromagnetic flow probe techniques are displayed in Table $\mathrm{I}$. Because the APDA supplies both pancreas and duodenum, microsphere data is broken down to exclude and include the parapancreatic duodenal segment. Regardless of the degree of arterial inflow occlusion, pancreatic blood flow determined by the microsphere method was significantly less than APDA flow measured by the electromagnetic probe method. The summed total pancreatic and parapancreatic duodenal segment blood flows measured by the microsphere method were also significantly less than APDA flows measured by the electromagnetic probe method.

\section{DISCUSSION}

Accurate measurement of pancreatic blood flow can be crucial in evaluating physiologic responses to various hormone, peptide, and pharmacologic agents and in assessing pathophysiologic responses to certain disease processes. Measurement of pancreatic blood flow can be difficult because the gland is not supplied entirely by a single arterial inflow vessel but rather is tripartite with contributions from the anterior pancreaticoduodenal artery, the posterior pancreaticoduodenal artery, and one or several branches from the splenic artery. Venous drainage is also tripartite and is compartmentalized, with little collateral between the areas of drainage [7].

Prior to the availability of radiolabeled carbonized plastic microspheres, total pan-

TABLE 1

Comparison of Methods of Measurement of PanCreatic Blood Flow in Milliters Per MinUte

\begin{tabular}{llcc}
\hline & \multicolumn{2}{c}{ Microsphere method } & \\
\cline { 2 - 3 } & Pancreas & $\begin{array}{c}\text { Pancreas and } \\
\text { duodenum }\end{array}$ & $\begin{array}{c}\text { Electromagnetic } \\
\text { probe method }\end{array}$ \\
\hline Intact inflow & $23 \pm 8^{*}$ & $38 \pm 10^{*}$ & $86 \pm 17$ \\
Occluded SA and PPDA & $19 \pm 4^{*}$ & $30 \pm 5^{*}$ & $94 \pm 27$ \\
\hline
\end{tabular}

* $P<0.05$, microsphere vs electromagnetic probe methods. 
creatic blood flow was measured in a variety of ways. One of the earliest methods employed was measuring the rate of venous drainage into a container [8]. This method alters venous outflow-pressure relationships and may interrupt neural and hormonally mediated physiologic pathways. Except for isolated pancreatic perfusion studies, this method has limited application.

Although measurement of pancreatic uptake of ${ }^{42} \mathrm{KCl}$ or ${ }^{86} \mathrm{RbCl}$ can be used to determine pancreatic blood flow and distribution [9-12], major methodological problems and restrictions limit the utility of the method. The most serious limitation involves the requirement for animal sacrifice at $60 \mathrm{sec}$ after intravenous injection of the isotope, eliminating the possibility of repeated interval blood flow determinations.

Other methods of organ blood flow measurement which might be applied to the pancreas include measurement of the rate of disappearance of a radioactive inert gas $\left({ }^{133} \mathrm{Xe}\right.$, ${ }^{85} \mathrm{Kr}$ ) which has been rapidly and specifically delivered to the pancreas [13] and measurement of the change in electrical potential measured with a platinum electrode during the inhalation of a hydrogen gas mixture [14]. However, these techniques have significant methodologic limitations in measurement of total pancreatic blood flow.

Measurement of canine pancreatic blood flow using electromagnetic flow probes has been attempted by several investigators [ $1-3$, 15]. The main advantages of this method are that it allows continuous flow measurement and obviates the need for animal sacrifice or removal of the pancreas for sampling. Organ anatomy is not distorted, the microcirculation is generally not affected, and the disease state of the pancreas does not alter the accuracy of measurement. Further, organ blood flow measurement with this technique is independent of simultaneous measurement of cardiac output and handling of isotopes can be avoided. Potential disadvantages are that the method is invasive and requires operative placement of flow probes, that operative dis- section may disrupt neuroregulatory pathways controlling the microcirculation, and that the tripartite arterial inflow of the canine pancreas might require the placement of three flow probes.

In actual use investigators have usually based the entire pancreas on the APDA, placing a single flow probe on the gastroduodenal artery, ligating the right gastroepiploic artery, the PPDA, and the pancreatic branches of the SA [1-3]. The validity of this technique is based on two assumptions: (1) the APDA is of sufficient size that it will not limit flow itself when the area of its distribution is increased and (2) there is no significant compartmentalization of the arterial inflow regions within the pancreas.

Neither of these assumptions have been adequately addressed in the literature. A preliminary study by Eichelter and Schenk [3], in which radiocontrast was injected into the APDA after ligation of all other pancreatic arterial inflow vessels, led to the conclusion that blood flow through the APDA adequately supplied the entire pancreas. However, the photograph accompanying their text did not delineate the borders of the pancreas, and they failed to demonstrate uniform perfusion of all portions of the gland, leaving their conclusion open to question. Contradictory evidence exists to suggest that arterial collateral within the canine pancreas is not adequate to support the gland when its arterial supply is based on the APDA alone. Babkin and Starling [4] noted that only the central portion of the canine pancreas remained viable during ex vivo perfusion when organ arterial blood flow was based solely on the APDA. Fischer et al. [5] maintained that slightly less than $60 \%$ of the canine pancreatic mass was perfused through the APDA after observing the distribution of India ink injected into the APDA.

Measurement of pancreatic blood flow using radiolabeled carbonized plastic microspheres addresses the assumptions made in measuring pancreatic blood flow with a single electromagnetic flow probe. The micro- 
sphere technique is well established and reliable for flow measurement, particularly if a "dummy organ" is used as a reference. In the dog, mixing of microspheres after left ventricular injection has been shown to be little different than after left atrial injection in measurement of blood flow to organs other than the heart [16]. Small $(15 \mu \mathrm{m})$ microspheres were desirable in our experiment because they distribute more like red blood cells and have proved more reliable in delineating regional blood flow [16].

In this experiment regional pancreatic blood flow studies showed an even distribution throughout the gland with intact tripartite arterial inflow. This observation was not altered by basing the pancreatic blood supply solely on the APDA, suggesting that adequate arterial collaterals exist to maintain perfusion throughout the gland, provided cardiac output and systemic arterial pressure are maintained. The loss of viability of the uncinate tip and the distal body and tail observed by Babkin and Starling [4] in an ex vivo perfused pancreas preparation was likely due to alterations in arterial flow or pressure, temperature, or intrapancreatic neurovascular regulation. Fischer's distribution study [5] can be questioned because of the distal site of injection of the India ink and the possibility of vascular reaction to such a foreign substance.

In this study, a difference of $71 \mathrm{ml} / \mathrm{min}$ (309\%) was determined between pancreatic blood flow measured by the microsphere method (SA and PPDA unclamped) versus that determined by the electromagnetic flow probe method (APDA flow, with SA and PPDA clamped) (Table I). Values for total pancreatic blood flow reported by other investigators, where measured by an electromagnetic flow probe on the APDA, have tended to be higher than those obtained by techniques in which pancreatic-specific flow was measured. Using a flow probe on the APDA, Eichelter and Schenk [3] measured blood flows ranging from 12 to $33.5 \mathrm{ml} / \mathrm{min}$. However, using the same model, Donaldson et al. [1] reported blood flows ranging from 44 to $49 \mathrm{ml} / \mathrm{min}$. In previous work in which the body and tail of the pancreas were removed, we observed baseline electromagnetic probe values of APDA flow that ranged from 43 to $63 \mathrm{ml} / \mathrm{min}$ [15]. Where attempts have been made to measure pancreatic-specific flow, the observed values were consistently lower. These latter methods include distribution of ${ }^{86} \mathrm{Rb}(14.9$ to $22.8 \mathrm{ml} / \mathrm{min})$ [9-11], anterior pancreaticoduodenal vein drainage ( $22 \mathrm{ml} / \mathrm{min}$ ) [8], and tracer microsphere blood flow measurement methods (14 $\mathrm{ml} / \mathrm{min}$, converted from $35 \mathrm{ml} / \mathrm{min} / 100 \mathrm{~g}$ ) [17]. Values obtained in this experiment for blood flow measured by an electromagnetic probe on the APDA compare favorably with values obtained by other investigators, while those obtained by the microsphere method were consistent with determinations made by others for pancreatic-specific flow.

At least two factors may be responsible for the discrepancy between methods. The first is that the flow probe method measures APDA flow, which distributes to the duodenum as well as to the pancreas. Delaney and Custer [9] reported canine duodenal blood flows of approximately $70 \mathrm{ml} / \mathrm{min} / 100 \mathrm{~g}$, a value comparable to our findings. The proportion of APDA flow that distributes to the duodenum has not been reported in the literature, and only the minimum proportion can be extracted from our data. After occlusion of the PPDA, blood flow to the parapancreatic duodenum (in this study, the proximal duodenum to the papilla of Vater) can be attributed to the APDA. Parapancreatic duodenal blood flow was $11 \mathrm{ml} / \mathrm{min}$ (Table 1), 37\% of combined pancreaticoduodenal flow. If APDA flow were limited to that short segment of parapancreatic duodenum, the duodenal proportion of APDA flow would be $37 \%$, and pancreatic blood flow derived from APDA flow would be 0.63 $\times 94 \mathrm{ml} / \mathrm{min}=59 \mathrm{ml} / \mathrm{min}$.

However, the APDA is likely to supply a much longer segment of proximal duodenum after the PPDA is occluded, to at least 
the extent of the uncinate. The length of the head-uncinate pancreatic segment $(22.2 \mathrm{~cm})$ was 2.8 times that of the parapancreatic duodenal segment $(7.9 \mathrm{~cm})$. If the assumption is made that the duodenum, extending the length of the head-uncinate segment, has weight and blood flow similar to those of the defined parapancreatic segment, duodenal blood flow to the larger segment would be $30.8 \mathrm{ml} / \mathrm{min}$. With these assumptions, "microsphere-determined" pancreaticoduodenal blood flow is $50 \mathrm{ml} / \mathrm{min}$, with a duodenal proportion of that flow equal to $62 \%$, and calculated pancreatic proportion of APDA flow would be $0.38 \times 94 \mathrm{ml} / \mathrm{min}=35 \mathrm{ml} /$ min. However, there remains a $16 \mathrm{ml} / \mathrm{min}$ (84\%) difference between methods of measurement.

A second factor that may contribute to the higher flows observed with the flow probe method is an inherent systematic error. Positioning the probe to maintain right-angle orientation to blood flow and good electrode contact and to avert angulation of the vessel is critical for accuracy of measurement. However, proper positioning is extremely difficult in vivo when continuous exposure of the vessel is to be avoided, particularly on small vessels. The tendency of the investigator is to adjust the position of the probe to achieve the highest flow value that is stable and internally consistent within the experiment while avoiding obvious angulation of the vessel. Unfortunately, without an immediate independent method of verifying the flow values, a systematic error toward high flows is likely to occur.

For measurement of canine pancreatic blood flow where absolute values of blood flow or intrapancreatic regional flows are of interest, a technique other than electromagnetic flow probe measurement of APDA flow should be utilized. Depending on the exigencies of the experiment, the radiolabeled microsphere technique should be strongly considered as it permits determination of both total and regional flows. Alternatives with strengths in measurement of regional flow and for more than six repeated measurements are radioactive inert gas washout and hydrogen gas washout techniques. In experiments where the change in pancreatic blood flow is the parameter of interest, measurement of APDA flow with an electromagnetic flow probe with the PPDA and the pancreatic branches of the SA occluded may be acceptable, but the factor of duodenal flow must be considered. No single method is suitable to all experimental situations; the information necessary for success of the study and the limitations of each method must be carefully weighed in choosing a technique for measurement of pancreatic blood flow.

\section{ACKNOWLEDGMENTS}

This work was supported by the Veterans Administration Research Service and the University of Michigan Computer Support Fund.

\section{REFERENCES}

1. Donaldson, L. A., Williams, R. W., and Schenk, W. G., Jr. Experimental pancreatitis: Effect of plasma and dextran on pancreatic blood flow. Surgery 84: 313, 1978.

2. Donaldson, L. A., and Schenk, W. G., Jr. Experimental acute pancrcatitis: The changes in pancreatic oxygen consumption and the effect of dextran 40 . Ann. Surg. 190: 728, 1979.

3. Eichelter, P., and Schenk, W. G., Jr. Hemodynamics of pancreatic secretion. Arch. Surg. 93: 200, 1966.

4. Babkin, B. P., and Starling, E. H. A method for the study of the perfused pancreas. J. Physiol. (London) 61: $245,1926$.

5. Fischer, U., Hommel, H., Jutzi, E., and Fiedler, $\mathbf{H}$. Modell der Netto-Insulinabgabe des Pankreas in vivo. Acta Biol. Med. Ger. 33: 21, 1974.

6. Heymann, M. A., Payne, B. D., Hoffman, J. I. E., and Rudolph, A. M. Blood flow measurements with radionuclide-labeled particles. Prog. Cardiovasc. Dis. 20: 55, 1977.

7. Kim, J. P., and Byrne, J. J. Segmental venous drainage of the canine pancreas. J. Surg. Res. 11: 559, 1971.

8. Pissiotis, C. A., Condon, R. E., and Nyhus, L. M. Effect of vasopressin on pancreatic blood flow in acute hemorrhagic pancreatitis. Amer. J. Surg. 123: 203, 1972. 
9. Delaney, J. P., and Custer, J. Gastrointestinal blood flow in the dog. Circ. Res. 17: 394, 1965.

10. Goodhead, B. Acute pancreatitis and pancreatic blood flow. Surg. Gynecol. Obstet. 129: 331, 1969.

11. Papp, M., Makara, G. B., Hajtman, B., and Csar, L. A quantitative study of pancreatic blood flow in experimental pancreatitis. Gastroenterology 51: $524,1966$.

12. Sapirstein, L. A. Regional blood flow by fractional distribution of indicators. Amer. J. Physiol. 193: $161,1958$.

13. Mackenzie, R. J., and Lieberman, D. P., et al. Liver blood flow measurement: The interpretation of xenon-133 clearance curves. Acta Chir. Scand. 142: $519,1976$.

14. Aune, S., and Semb, L. S. The effect of secretin and pancreozymin on pancreatic blood flow in the conscious and anesthetized dog. Acta Physiol. Scand. 76: 406, 1969.

15. Knol, J. A., Edgcomb, L. P., Inman, M. G., and Eckhauser, F. E. Low molecular weight dextran in experimental pancreatitis: Effects on pancreatic microcirculation. J. Surg. Res. 35: 73, 1983.

16. Buckberg, G. D., Luck, J. C., Payne, D. B., Hoffman, J. I. E., Archie, J. P., and Fixler, D. F. Some sources of error in measuring regional blood flow with radioactive microspheres. J. Appl. Physiol. 31: 598, 1971.

17. Becker, H., Vinten-Johansen, J., Buckberg, G. D., and Bugyi, H. I. Correlation of pancreatic blood flow and high-energy phosphates during experimental pancreatitis. Eur. Surg. Res. 14: 203, 1982. 